\title{
Faint open clusters with 2MASS: BH 63, Lyngå 2, Lyngå 12 and King 20
}

\author{
E. Bica, C. Bonatto, and R. Blumberg
}

\begin{abstract}
Universidade Federal do Rio Grande do Sul, Instituto de Física, CP 15051, Porto Alegre 91501-970, RS, Brazil
e-mail: [charles; bica; blumberg]@if.ufrgs.br
\end{abstract}

Received 17 April 2006 / Accepted 4 September 2006

\section{ABSTRACT}

\begin{abstract}
Context. Structural and dynamical parameters of faint open clusters are probed with quality 2MASS-photometry and analytical procedures developed for bright clusters.

Aims. We derive fundamental parameters of the faint open clusters Lyngå 2, BH 63, Lyngå 12 and King 20, the last three of which have no prior determinations. We also focus on the structure and dynamical state of these clusters.

Methods. $J, H$ and $K_{\mathrm{s}} 2$ MASS photometry with errors smaller than 0.2 mag are used to build CMDs, radial density profiles, colourcolour diagrams, luminosity and mass functions. Colour-magnitude filters are used to isolate probable member stars. Field-star decontamination is applied to Lyngå 2, Lyngå 12 and King 20 .

Results. Reddening values are in the range $0.22 \leq E(B-V) \leq 1.9$, with BH 63 the most reddened object. Ages of Lyngå 2, King 20, Lynga 12 and BH 63 are $\approx 90, \approx 200, \approx 560$ and $\approx 700$ Myr, respectively. The radial density distributions of Lyngå 12 and King 20 are well-represented by King profiles. Lyngå 2 and BH 63 are very small with core and limiting radii of $\approx 0.12 \mathrm{pc}$ and $\approx 1.5 \mathrm{pc}$. Yet, they fit in the small-radii tail of the open cluster size distribution. Lyngå 12 and King 20 have $R_{\text {core }} \approx 0.43 \mathrm{pc}$ and $R_{\lim } \approx 3.9$ pc. Lyngå 2 and Lyngå 12 are inside the Solar circle. Total stellar masses (extrapolating the MFs to stars with $0.08 M_{\odot}$ ) range from $\approx 340 M_{\odot}$ (BH 63) to $\approx 2300 M_{\odot}$ (Lynga 12). Observed masses are $\sim 1 / 4$ of these values. In all clusters the core mass function is flatter than the halo's. Conclusions. Faint open clusters can be probed with 2MASS when associated with colour-magnitude filters and field-star decontamination. BH 63 appears to be in an advanced dynamical state, both in the core and halo. To a lesser degree the same applies to King 20. Marginal evidence of dynamical evolution is present in the cores of Lyngå 2 and Lyngå 12.
\end{abstract}

Key words. open clusters and associations: general - open clusters and associations: individual: BH63 - open clusters and associations: individual: Lyngå 2 - open clusters and associations: individual: Lyngå 12 - open clusters and associations: individual: King 20 - Galaxy: structure

\section{Introduction}

Because it is relatively simple to estimate ages and distances of open clusters (OCs), they have become fundamental probes of Galactic disc properties (Lyngå 1982; Janes \& Phelps 1981; Friel 1995; Bonatto et al. 2006a; Piskunov et al. 2006). However, the proximity of most of the OCs to the plane and the corresponding high values of reddening and field-star contamination (Bonatto et al. 2006a, and references therein) usually restrict this analysis to the more populous OCs and/or those located a few kpc from the Sun.

To probe disc structure Bonatto et al. (2006a) employed 654 OCs with fundamental parameters (such as age, distance from the Sun, and reddening) mostly from the WEBDA ${ }^{1}$ database (Mermilliod 1996). They found that a large fraction of the faint and/or poorly populated OCs must be overwhelmed in the field, particularly in bulge/disc directions. Piskunov et al. (2006) used 650 OCs from ASCC-2.5 data to infer kinematical similarities among OC groups. Both works estimate a total population of $\sim 10^{5}$ OCs in the Galaxy. Dias et al. (2002) reported 1756 optically visible OCs and candidates in their catalogue. These works suggest that besides those not yet observed, there is a large number of OCs and candidates whose properties have not been explored.

\footnotetext{
${ }^{1}$ http://obswww. unige.ch/webda
}

Efforts to analyse unstudied OCs and derive their fundamental parameters will contribute to future disc studies by unveiling the properties of individual OCs themselves and constraining theories of molecular cloud fragmentation, star formation and stellar and dynamical evolution. These processes will be better understood as the number of studied clusters, faint ones in particular, increases. A side-product of more constrained parameters will be better statistics of OCs as a class.

As a first step in this direction we studied a series of faint OCs (Bica et al. 2003; Bonatto et al. 2004; Bica \& Bonatto 2005) using near-IR photometry. For spatial and photometric uniformity we work with $J, H$ and $K_{\mathrm{s}} 2 \mathrm{MASS}^{2}$ Point Source Catalogue (PSC) photometry. We have developed methods to disentangle cluster and field stars in colour-magnitude diagrams (CMDs), as well as in radial stellar density distributions. The use of field-star decontamination and colour-magnitude filters (hereafter C-MF) have produced more robust parameters (e.g. Bonatto \& Bica 2005; Bonatto et al. 2006b). In particular, field-star decontamination constrains more the age, especially for low-latitude OCs (Bonatto et al. 2006a). These procedures have proven useful also in the analysis of faint and/or distant OCs (Bica et al. 2003; Bonatto et al. 2004; Bica \& Bonatto 2005). Recently we

\footnotetext{
2 The Two Micron All Sky Survey, All Sky data release (Skrutskie et al. 1997), available at http://www.ipac.caltech.edu/2mass/ releases/allsky/
} 
discussed the advantages of using C-MFs in the construction of radial density profiles for M 52 and NGC 3960, whose fields are affected by different amounts of differential reddening (Bonatto $\&$ Bica 2006). As by-products we have been deriving more constrained structural parameters such as core and limiting radii, and mass function slopes, that allow inferences on dynamical evolution.

In the present work we focus on the faint OCs Lyngå 2, Lyngå 12, King 20 (Alter et al. 1970, and references therein), and BH 63 that was catalogued by van den Bergh \& Hagen (1975).

We intend to derive photometric, cluster structure and dynamic-related parameters working with a set of methods which provide more accurate results. The target clusters are Lyngå 2 (for comparison purposes with the literature), and the as yet unstudied OCs Lyngå 12, King 20 and BH 63. Since BH 63 is very reddened, and consequently differential reddening may affect its field, it required an approach slightly different than those for the other OCs. Throughout this paper errors correspond to $1 \sigma$ Poisson fluctuations.

This paper is organized as follows. In Sect. 2 we discuss basic properties and review literature data (when available) on the 4 clusters. In Sect. 3 we present the 2MASS data, subtract the field-star contamination (except for BH 63), derive fundamental cluster parameters, and analyse the radial density profiles. In Sect. 4 we derive luminosity and mass functions (LFs and MFs), and compute stellar content properties. In Sect. 5 we discuss dynamical states. Concluding remarks are given in Sect. 6.

\section{The target open clusters}

Ruprecht (1966) classified Lyngå 2 and Lyngå 12 as poor loose OCs of Trumpler types IV2p and IV1p, respectively. King 20 was considered somewhat more concentrated with type II1p. The only previously studied object is Lyngå 2 . Based on the photographic UBV analysis by Lindoff (1968), WEBDA provides a reddening $E(B-V)=0.20$, distance from the Sun $d_{\odot}=1 \mathrm{kpc}$ and age $\approx 130 \mathrm{Myr}$. Kharchenko et al. (2005) found $E(B-V)=0.20, d_{\odot}=1 \mathrm{kpc}$ and age $\approx 260 \mathrm{Myr}$. They also estimated a core radius of $4.8^{\prime}$ and a cluster radius of 13.2'.

In Fig. 1 we show XDSS ${ }^{3} \mathrm{R}$ images of BH 63 (left panel) and Lyngå 2 (right panel). In Fig. 2 we present the images of Lyngå 12 (left panel) and King 20 (right panel).

The clusters are projected close to the plane (Table 1). Nevertheless their fields are differently populated owing to their Galactic longitudes. Lyngå 12 and Lyngå 2 are located in the 4th quadrant, and Lyngå 12 is probably contaminated by bulge stars (Sect. 3.1). They present low stellar density contrast as expected from their Trumpler classifications. King 20 is in the 2nd quadrant and appears looser than a Trumpler type II cluster. Probably the best classification would be a type III. BH 63, located near the border between the 3rd and 4th quadrants, has a significant contrast with respect to the field. It is projected in the direction of the dark nebula FeSt 1-108 (Feitzinger \& Stüwe 1984; Dutra $\&$ Bica 2002) with an angular diameter of $96^{\prime}$. The high reddening of BH 63 (Sect. 3.3) suggests that the nebula is located in the cluster foreground. No velocity is available for the nebula, so a distance estimate is not available, but the advanced dynamical state of BH 63 (Sect. 5) might be related to an interaction with a molecular cloud. More detailed studies are necessary to verify this possibility.

\footnotetext{
${ }^{3}$ Second Generation Digitized Sky Survey, extracted from the Canadian Astronomy Data Centre (CADC), at http://cadcwww. dao.nrc.ca/
}

In Table 1 we provide data on the clusters. Right ascension, declination and diameter (Cols. 2 to 4 ) were measured visually by us on XDSS images (Figs. 1 and 2) as the best approximation of the cluster center. However, the stellar radial density profiles (Sect. 3.4) presented a dip at these centers in all clusters. Consequently, we searched for new coordinates that would maximize the central density of stars by examining histograms for the number of stars in $0.5^{\prime}$ bins of right ascension and declination on C-MF photometry (Sect. 3.4). The new central coordinates and the corresponding Galactic longitude and latitude are given in Cols. 5 to 8 of Table 1. Age, central reddening, distance from the Sun and Galactocentric distance based on 2MASS (Sect. 3.3) are in Cols. 9 to 13. Alternative cluster names are in Col. 14.

\section{The 2MASS photometry}

VizieR $^{4}$ was used to extract $J, H$ and $K_{\mathrm{s}}$ 2MASS photometry in a circular area centered on the optimized coordinates of the 4 clusters (Cols. 5 and 6 of Table 1). Extraction radii are $R=20^{\prime}$ (BH 63), $R=35^{\prime}$ (Lyngå2), and $R=40^{\prime}$ (Lyngå 12 and King 20). Our previous experience (Sect. 1) shows that as long as no other cluster is present in the field, such large extraction areas provide the required statistics for field-star characterisation. For photometric quality constraints the extraction was restricted to stars (i) brighter than the $99.9 \%$ Point Source Catalogue Completeness Limit ${ }^{5}, J=15.8, H=15.1$ and $K_{\mathrm{s}}=14.3$, respectively; and (ii) with errors in $J, H$ and $K_{\mathrm{s}}$ smaller than $0.2 \mathrm{mag}$. For reddening transformations we use the relations $A_{J} / A_{V}=0.276, A_{H} / A_{V}=0.176, A_{K_{\mathrm{S}}} / A_{V}=0.118$, and $A_{J}=2.76 \times E(J-H)$ (Dutra et al. 2002), assuming a constant total-to-selective absorption ratio $R_{V}=3.1$.

$J \times(J-H)$ CMDs of regions that provide a suitable contrast with the field are shown in Fig. 3 (left panels). For BH 63 we extracted photometry from a region with $R=2.5^{\prime}$ centered on the optimized coordinates (Table 1). For Lyngå 2, Lyngå 12 and King 20 we used $R=5^{\prime}$. The extension and morphology of the main sequences (MS) indicate that cluster age increases from Lyngå 2, King 20, Lyngå 12 and BH 63. Because of the lowlatitudes, field stars (mostly disc) contaminate the CMDs particularly at faint magnitudes and red colours. Bulge stars (mostly red giants) contaminate the field of Lyngå 12 .

\subsection{Field-star decontamination}

Field stars are usually an important contaminant of CMDs, particularly of low-latitude OCs and/or those projected against the bulge. Their presence in the fields of the target clusters can be seen in the corresponding offset-field CMDs (middle panels of Fig. 3), extracted from a region outside each cluster's limiting radius (Sect. 3.4) with the same projected area as the central extraction (left panels of Fig. 3).

To infer the intrinsic cluster-CMD morphology we use the field-star decontamination procedure previously applied in the analysis of low-contrast (Bica \& Bonatto 2005), young embedded (Bonatto et al. 2006), and young (Bonatto et al. 2006b) OCs. The algorithm works on a statistical basis that takes into account the relative densities of stars in a cluster region and offset field. In short; it (i) computes the surface density of field stars (according

\footnotetext{
4 http://vizier.u-strasbg.fr/viz-bin/ VizieR?-source=II/246

5 Following the Level 1 Requirement, according to http: //www . ipac. caltech. edu/2mass/releases/ allsky/doc/sec6_5a1.html
} 


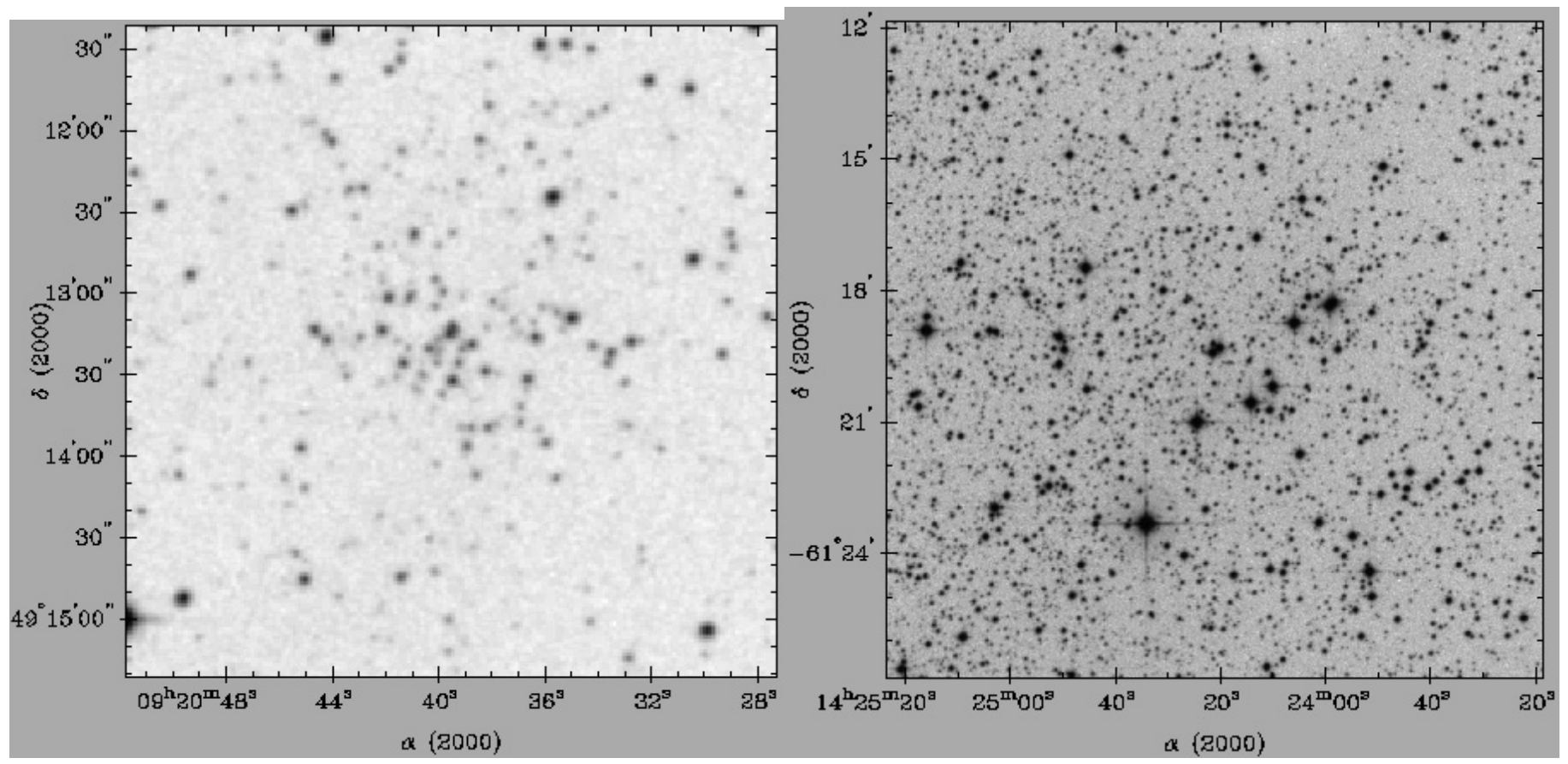

Fig. 1. Left panel: $4^{\prime} \times 4^{\prime}$ XDSS R image of BH 63. Right panel: $15^{\prime} \times 15^{\prime}$ XDSS R image of Lyngå 2. Images centered on the optimised coordinates (Cols. 5 and 6 of Table 1).
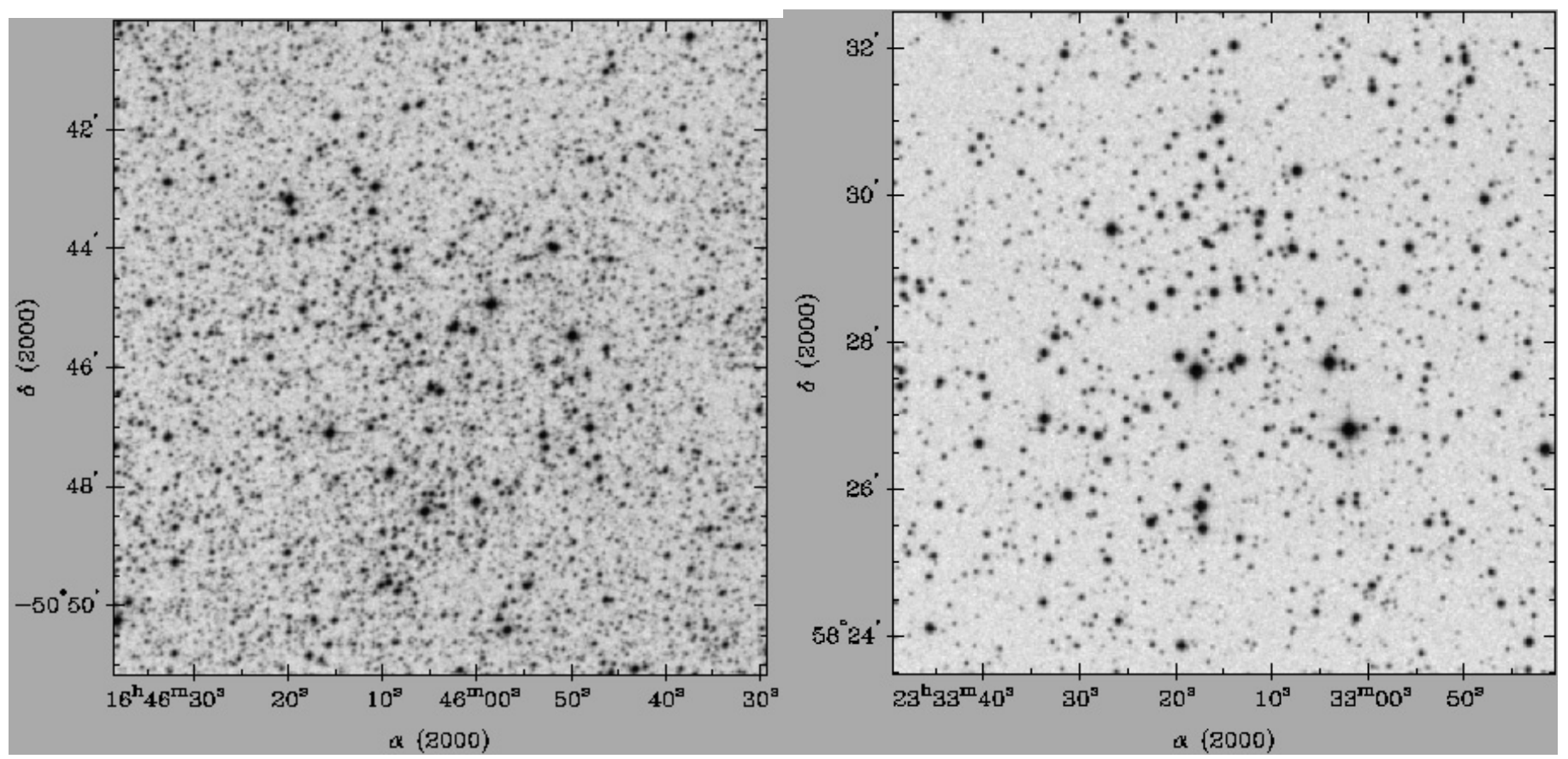

Fig. 2. Same as Fig. 1 for the $11^{\prime} \times 11^{\prime}$ field around Lyngå 12 (left panel) and the $9^{\prime} \times 9^{\prime}$ field around King 20 (right panel).

to the number of stars in the respective offset field); (ii) assumes that it is uniform throughout the cluster field; (iii) divides the CMD in colour/magnitude cells of varying size; (iv) randomly assigns which star within a CMD cell will be assumed to be a field star; and finally (v) subtracts the expected number of candidate field stars from each cell. The size of the colour/magnitude cells can be subsequently adjusted so that the total number of subtracted stars (summing up all cells) matches the expected number of field stars. Because the remaining stars are in CMD cells where the stellar density presents a clear excess over the field, they have a high probability of being cluster members. In crowded field regions, field-star density at faint magnitudes (usually $J \geq 14.5$ in 2MASS) may be equal or even larger than that expected from the cluster. In such cases the present decontamination procedure would produce an artificially truncated MS at the sub-solar mass range, even for old OCs. Further details on this procedure are in Bonatto et al. (2006b). As offset fields we take the regions $20 \leq R\left({ }^{\prime}\right) \leq 35$ (Lyngå2), $25 \leq R\left({ }^{\prime}\right) \leq 40$ (Lyngå 12), and $20 \leq R\left(\left(^{\prime}\right) \leq 40\right.$ (King 20), that are large enough to produce field-star statistical representativity, both in magnitude and colours. This procedure could not be applied to BH 63 because of the high reddening and small angular size (Sects. 3.3 
Table 1. General data on the clusters.

\begin{tabular}{|c|c|c|c|c|c|c|c|c|c|c|c|c|c|}
\hline \multirow[b]{2}{*}{ Cluster } & \multicolumn{3}{|c|}{ Derived from XDSS } & \multicolumn{9}{|c|}{ Derived from 2MASS } & \multirow[b]{2}{*}{$\begin{array}{c}\text { Alternative } \\
\text { names }\end{array}$} \\
\hline & $\begin{array}{c}\alpha(2000) \\
(\mathrm{hms})\end{array}$ & $\begin{array}{c}\delta(2000) \\
\left({ }^{\prime \prime \prime \prime}\right)\end{array}$ & $\begin{array}{l}D \\
(')\end{array}$ & $\begin{array}{c}\alpha(2000) \\
(\mathrm{hms})\end{array}$ & $\begin{array}{c}\delta(2000) \\
\left({ }^{\circ} \prime^{\prime \prime}\right)\end{array}$ & $\begin{array}{c}\ell \\
\left(^{\circ}\right)\end{array}$ & $\begin{array}{l}b \\
\left({ }^{\circ}\right)\end{array}$ & $\begin{array}{c}\text { Age } \\
(\mathrm{Myr})\end{array}$ & $E(B-V)$ & $\begin{array}{c}d_{\odot} \\
(\mathrm{kpc})\end{array}$ & $\begin{array}{c}d_{\mathrm{GC}} \\
(\mathrm{kpc})\end{array}$ & $\begin{array}{c}d_{\mathrm{GC}} \\
(\mathrm{kpc})\end{array}$ & \\
\hline (1) & (2) & (3) & (4) & (5) & (6) & (7) & (8) & (9) & (10) & (11) & (12) & (13) & (14) \\
\hline
\end{tabular}

\begin{tabular}{lllllllll}
\hline BH 63 & $09: 20: 39-49: 13: 23$ & 2.5 & $09: 20: 39.5$ & $-49: 13: 20.5$ & $271.63+0.40700 \pm 100$ & $1.9 \pm 0.6$ & $2.3 \pm 0.38 .3 \pm 0.37 .5 \pm 0.3$ & ESO $212 S C 2$
\end{tabular}

Lyngå 2 14:24:35 -61:19:50 13.0 14:24:21.1 -61:19:21.0 313.84 -0.43 90 $10 \quad 0.22 \pm 0.04$ 0.9 $0.17 .4 \pm 0.16 .6 \pm 0.1$ OCl-916, ESO 134SC2, BH 157 Lyngå 12 16:46:04 -50:45:50 $8.0 \quad 16: 46: 04.4-50: 45: 40.1335 .70-3.46560 \pm 1000.22 \pm 0.04 \quad 1.0 \pm 0.27 .1 \pm 0.26 .3 \pm 0.2 \quad$ OCl-973, ESO 226SC22

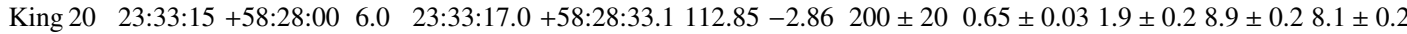

$\mathrm{OCl}-262$

Table notes: Cols. 2 and 3: measured by us on XDSS images; Col. 4: angular diameter estimated on XDSS images; Col. 10: reddening in the cluster's central region (Sect. 3.3); Col. 12: $d_{\mathrm{GC}}$ calculated using $R_{\mathrm{O}}=8.0 \mathrm{kpc}$ (Reid 1993) as the distance from the Sun to the Galactic center; Col. 13: $d_{\mathrm{GC}}$ using $R_{\mathrm{O}}=7.2 \mathrm{kpc}$ (Bica et al. 2006).

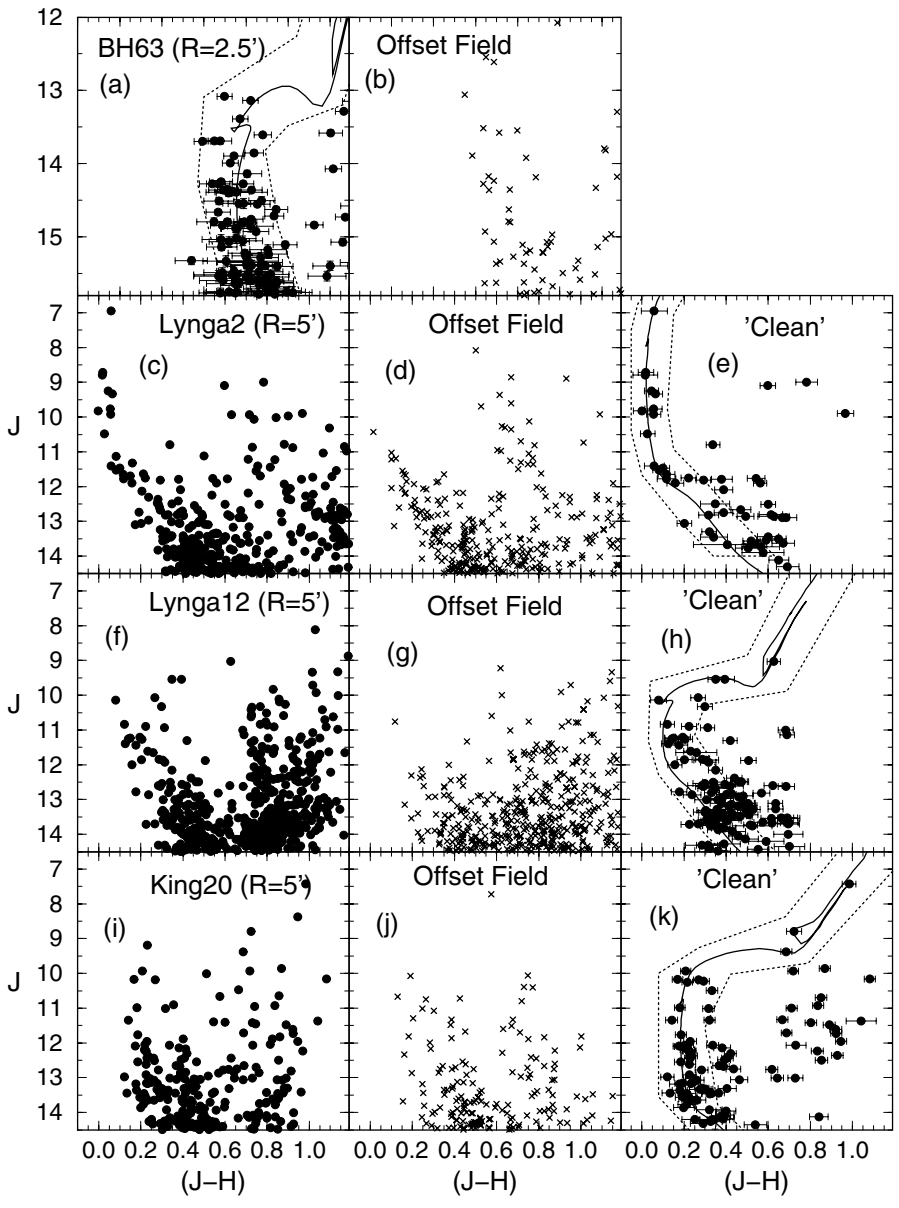

Fig. 3. Left panels: observed CMDs. Middle panels: field stars. Right panels: field-star decontaminated CMDs. The solid line in panels a), e), h), and k) shows the Padova isochrone, while the dotted line represents the C-MF used to isolate cluster MS/evolved stars. Field-star decontamination was not applied to $\mathrm{BH} 63$ because of high reddening.

and 3.4). Because it actually subtracts stars from the original files - thus artificially changing the radial distribution of stars - we use the field-star decontamination only to uncover the intrinsic cluster CMD morphologies and build colour-colour diagrams (Fig. 4). Cluster structure and luminosity/mass functions are analysed with the stars selected with the colour-magnitude filters shown in panels (a), (e), (h), and (k) of Fig. 3.

In Table 2 we summarise the results of the decontamination procedure in terms of the fraction of member and field stars
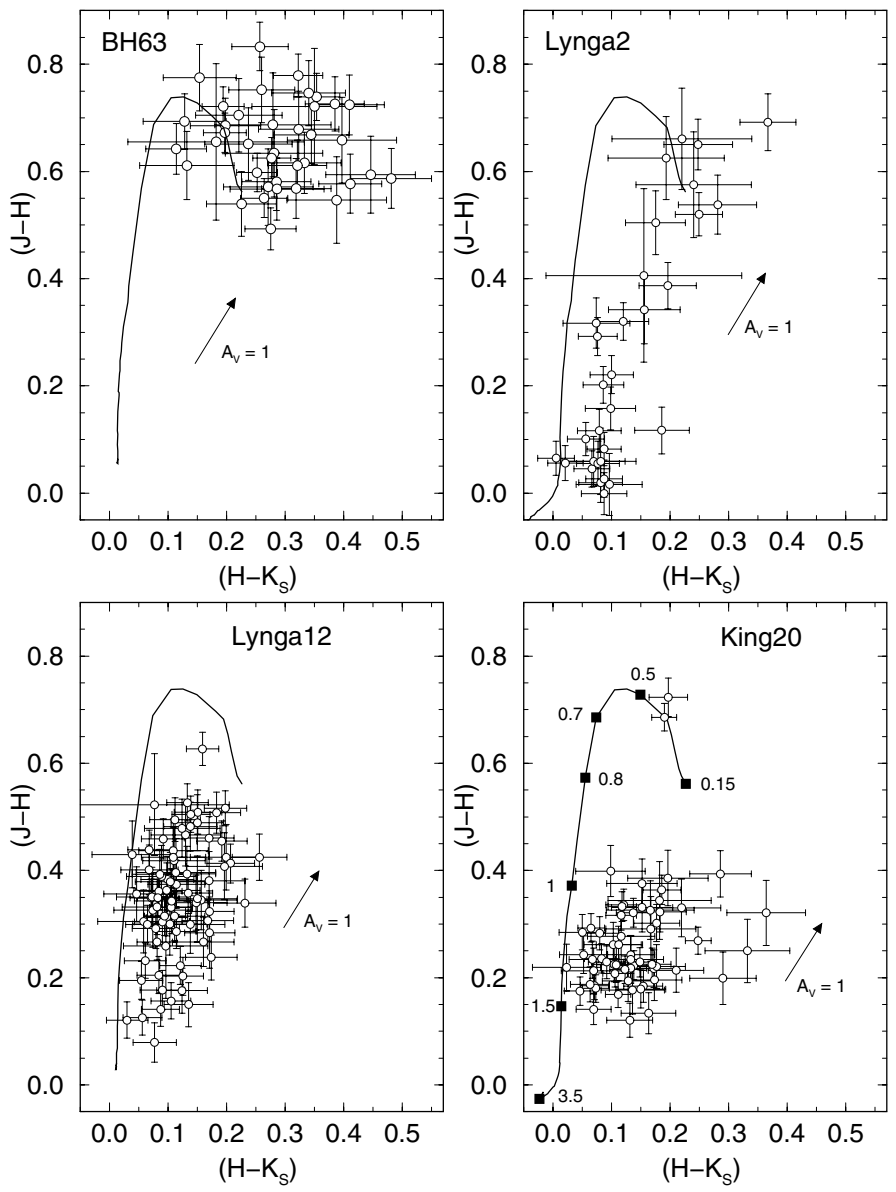

Fig. 4. Colour-colour diagrams. Solid line: MS range of the Padova isochrones of ages $700 \mathrm{Myr}$ (BH 63), $90 \mathrm{Myr}$ (Lyngå 2), $560 \mathrm{Myr}$ (Lyngå 12), and $200 \mathrm{Myr}$ (King 20). Arrows: reddening vectors $E(J-H)=1.72 \times E\left(H-K_{\mathrm{s}}\right)$ for $A_{V}=1$. For illustrative purposes, representative masses (in $M_{\odot}$ ) are indicated along the $200 \mathrm{Myr}$ isochrone (King 20).

throughout the fields of the target clusters. Member stars appear to be found up to $R \approx 4-6^{\prime}, R \approx 12-14^{\prime}$, and $R \approx 6-8^{\prime}$ from the centers of Lyngå 2 , Lyngå 12 and King 20 . Because of its nearbulge and central disc direction $\left(\ell \approx 336^{\circ}\right)$, the fraction of member stars in the field of Lyngå 12 is $\approx 1 / 3$ of that in the field of $\operatorname{King} 20\left(\ell \approx 113^{\circ}\right)$; for Lyngå $2\left(\ell \approx 314^{\circ}\right)$ it is intermediate between both. 
Table 2. Cluster and field-star statistics.

\begin{tabular}{|c|c|c|c|c|c|c|c|c|c|}
\hline \multirow[b]{2}{*}{$\begin{array}{l}\Delta R \\
\left(^{\prime}\right)\end{array}$} & \multicolumn{3}{|c|}{ Lyngå 2} & \multicolumn{3}{|c|}{ Lyngå 12} & \multicolumn{3}{|c|}{ King 20} \\
\hline & $\begin{array}{r}N_{\text {obs }} \\
\text { (stars) }\end{array}$ & $\begin{array}{r}f_{\mathrm{cl}} \\
(\%)\end{array}$ & $\begin{array}{r}f_{\mathrm{fs}} \\
(\%)\end{array}$ & $\begin{array}{r}N_{\text {obs }} \\
\text { (stars) }\end{array}$ & $\begin{array}{r}f_{\mathrm{cl}} \\
(\%)\end{array}$ & $\begin{array}{r}f_{\mathrm{fs}} \\
(\%)\end{array}$ & $\begin{array}{r}N_{\text {obs }} \\
\text { (stars) }\end{array}$ & $\begin{array}{r}f_{\mathrm{cl}} \\
(\%)\end{array}$ & $\begin{array}{r}f_{\mathrm{f}} \\
(\%)\end{array}$ \\
\hline (1) & (2) & (3) & (4) & (5) & (6) & (7) & (8) & (9) & $(10)$ \\
\hline $0-2$ & 80 & 34 & 66 & 167 & 32 & 68 & 77 & 44 & 56 \\
\hline $2-4$ & 1841 & 14 & 86 & 442 & 23 & 77 & 165 & 21 & 79 \\
\hline $4-6$ & 271 & 3 & 97 & 651 & 13 & 87 & 243 & 11 & 89 \\
\hline $6-8$ & 336 & 0 & 100 & 1152 & 7 & 93 & 315 & 4 & 96 \\
\hline $8-10$ & - & - & - & 1491 & 7 & 93 & 399 & 0 & 100 \\
\hline $10-12$ & - & - & - & 1765 & 4 & 96 & - & - & - \\
\hline $12-14$ & - & - & - & 2064 & 3 & 97 & - & - & 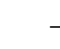 \\
\hline $14-16$ & - & - & - & 2312 & 0 & 100 & - & - & - \\
\hline Total & 451 & 12 & 88 & 6853 & 6 & $\overline{94}$ & 645 & 18 & 82 \\
\hline
\end{tabular}

Table notes: Cols. 2, 5 and 8: number of observed stars in the region; Cols. 3, 6 and 9: fraction of member stars; Cols. 4, 7 and 10: fraction of field stars. Total fields are $R=5.5^{\prime}$ (Lyngå 2), $R=13^{\prime}$ (Lyngå 12) and $R=7^{\prime}$ (King 20).

The field-star decontaminated CMDs of the $R \leq 5^{\prime}$ regions are shown in the right panels of Fig. 3, where error bars are included to show the magnitude of the photometric uncertainties. As expected, most of the red and faint stars were considered as field stars by the decontamination procedure.

\subsection{Colour-colour diagrams}

Colour-colour diagrams with near-IR photometry have been useful particularly in the analysis of young clusters, e.g. NGC 6611 (Bonatto et al. 2006), Trapezium and the cluster embedded in the nebula NGC 2327 (Soares \& Bica 2002). We show in Fig. 4 the $(J-H) \times\left(H-K_{\mathrm{s}}\right)$ diagrams of the target OCs together with the MS portion of the respective Padova isochrones (Sect. 3.3) and the reddening vector $(E(J-H)=$ $\left.1.72 \times E\left(H-K_{\mathrm{s}}\right)\right)$ for $A_{V}=1$. For membership considerations we used only the stars selected with the C-MFs applied to the field-star decontaminated photometry of Lyngå 2, Lyngå 12 and King 20 (panels e, $h$ and $k$ of Fig. 3). Because of the high reddening in the field of BH 63 (Sect. 3.1), the corresponding colourcolour diagram was produced with the stars selected after applying the colour-magnitude filter (panel (a) of Fig. 3). Most of the stars in Lyngå 2, Lyngå 12 and King 20 distribute along the MS of the Padova isochrones which suggests that reddening values in their fields are not exceedingly high. The locus and colourspread in $\mathrm{BH} 63$, on the other hand, reflect a high reddening and some differential reddening in the field.

Red stars around the $\mathrm{M}$ dwarf loci $((J-H) \approx 0.7$ and $\left.\left(H-K_{\mathrm{s}}\right) \approx 0.15\right)$ are probably residual field stars, since we cannot detect $\mathrm{M}$ dwarfs in these clusters with 2MASS. Particularly in Lyngå 2 and Lyngå 12 the red stars follow the reddening vector, again suggesting residual field contamination.

\subsection{Cluster age and distance from the Sun}

Cluster age is derived with solar-metallicity - which is typical of OCs (Bonatto et al. 2004a; WEBDA) - Padova isochrones (Girardi et al. 2002) computed with the 2MASS $J, H$ and $K_{\mathrm{s}}$ filters ${ }^{6}$ ones, with differences of at most 0.01 in $(J-H)$ (Bonatto et al. 2004a).

The field-star decontaminated CMD morphologies (right panels of Fig. 3) provide enough constraints to derive the cluster ages of Lyngå 2, Lyngå 12 and King 20. Particularly for Lyngå 2 and Lyngå 12, the field-star decontaminated CMDs provide unambiguous isochrone fits because of the conspicuous upper-MS in Lyngå 2 and the turnoff and giant branch in Lyngå 12 (panels (e) and (h) of Fig. 3). To a lesser degree, the same applies to King 20, in which the presence of 3 bright stars with $(J-H) \geq$ 0.65 and $J \leq 9.5$ suggest evolved stars (panel (k)). For Lyngå 2 we found an age of $90 \pm 10 \mathrm{Myr}$, observed distance modulus $(m-M)_{J}=9.9 \pm 0.1$ and colour excess $E(J-H)=0.07 \pm 0.01$, converting to $E(B-V)=0.22 \pm 0.03$. This age-solution is plotted in panel (e) of Fig. 3. The absolute distance modulus is $(m-M)_{\mathrm{O}}=9.7 \pm 0.1$, resulting in $d_{\odot}=0.9 \pm 0.1 \mathrm{kpc}$. These values are in good agreement with those in the literature (Sect. 2). The turnoff occurs at $M_{J} \approx-2.1$ and $m \approx 5.4 M_{\odot}$. Because of the 2MASS faint-magnitude limit (Sect. 3), MS stars are detected for $m \geq 0.9 M_{\odot}$. The Galactocentric distance of Lyngå 2 is $d_{\mathrm{GC}}=7.4 \pm 0.2 \mathrm{kpc}$, using $R_{\mathrm{O}}=8.0 \mathrm{kpc}$ as the Sun's distance to the Galactic center (Reid 1993). However, with the recently derived value of $R_{\mathrm{O}}=7.2 \mathrm{kpc}$ (based on updated parameters of globular clusters - Bica et al. 2006), this OC ends up located $d_{\mathrm{GC}}=6.6 \pm 0.1 \mathrm{kpc}$ from the Galactic center. In any case, Lyngå 2 is located $\approx 0.6 \mathrm{kpc}$ inside the Solar circle.

For Lyngå 12 we derived $E(J-H)=0.07 \pm 0.01$ $(E(B-V)=0.22 \pm 0.03)$, an age of $560 \pm 100 \mathrm{Myr}$ and $d_{\odot}=1.0 \pm 0.1 \mathrm{kpc}$ (panel $\left.\mathrm{h}\right) ; \mathrm{MS}$ stars are detected in the range $0.8 \leq m\left(M_{\odot}\right) \leq 2.5$. For King 20 we found $E(J-H)=$ $0.21 \pm 0.01(E(B-V)=0.65 \pm 0.03)$, an age of $200 \pm 20 \mathrm{Myr}$ and $d_{\odot}=1.9 \pm 0.2 \mathrm{kpc}$ (panel k). MS stars are detected in the range $1.2 \leq m\left(M_{\odot}\right) \leq 3.8$. Additional parameters are given in Table 1.

Parameters of BH 63 were based on the observed photometry. We found $E(J-H)=0.60 \pm 0.02(E(B-V)=1.9 \pm 0.6)$, age $=700 \pm 100 \mathrm{Myr}$ and $d_{\odot}=2.3 \pm 0.3 \mathrm{kpc}$; the detected MS mass range is $1.5 \leq m\left(M_{\odot}\right) \leq 2.3$ (panel (a)). BH 63 is a heavily reddened cluster, with $A_{V} \approx 6.1$.

\subsection{Cluster structure}

Cluster structure was inferred with the radial density profile (RDP), defined as the projected number-density of MS/evolved stars around the center. The RDPs were built with stars selected with the C-MFs (right panels of Fig. 3). The use of C-MFs to discard foreground/background field stars was applied in the analysis of the OCs M 67 (Bonatto \& Bica 2003), NGC 3680 (Bonatto et al. 2004b), NGC 188 (Bonatto et al. 2005), NGC 6611 (Bonatto et al. 2006), and NGC 4755 (Bonatto et al. 2006b). To avoid oversampling near the center and undersampling for large radii, the RDPs were built by counting stars in concentric rings with radius $\Delta R=0.5^{\prime}$ for $0 \leq R\left(^{\prime}\right)<5, \Delta R=1^{\prime}$ for $5 \leq R\left(^{\prime}\right)<10, \Delta R=2^{\prime}$ for $10 \leq R\left(^{\prime}\right)<30$ and $\Delta R=4^{\prime}$ for $R \geq 30^{\prime}$.

Figure 5 shows the RDPs for the MS/evolved stars of the present OCs. For absolute comparison between clusters the radius scale was converted to parsecs and the number-density of stars to stars $\mathrm{pc}^{-2}$ using the distances derived in Sect. 3.3.

\footnotetext{
6 http://pleiadi.pd.astro.it/isoc_photsys.01/ isoc_photsys.01.html. 2MASS transmission filters produced isochrones very similar to the Johnson-Kron-Cousins (e.g. Bessel \& Brett 1988).
} 

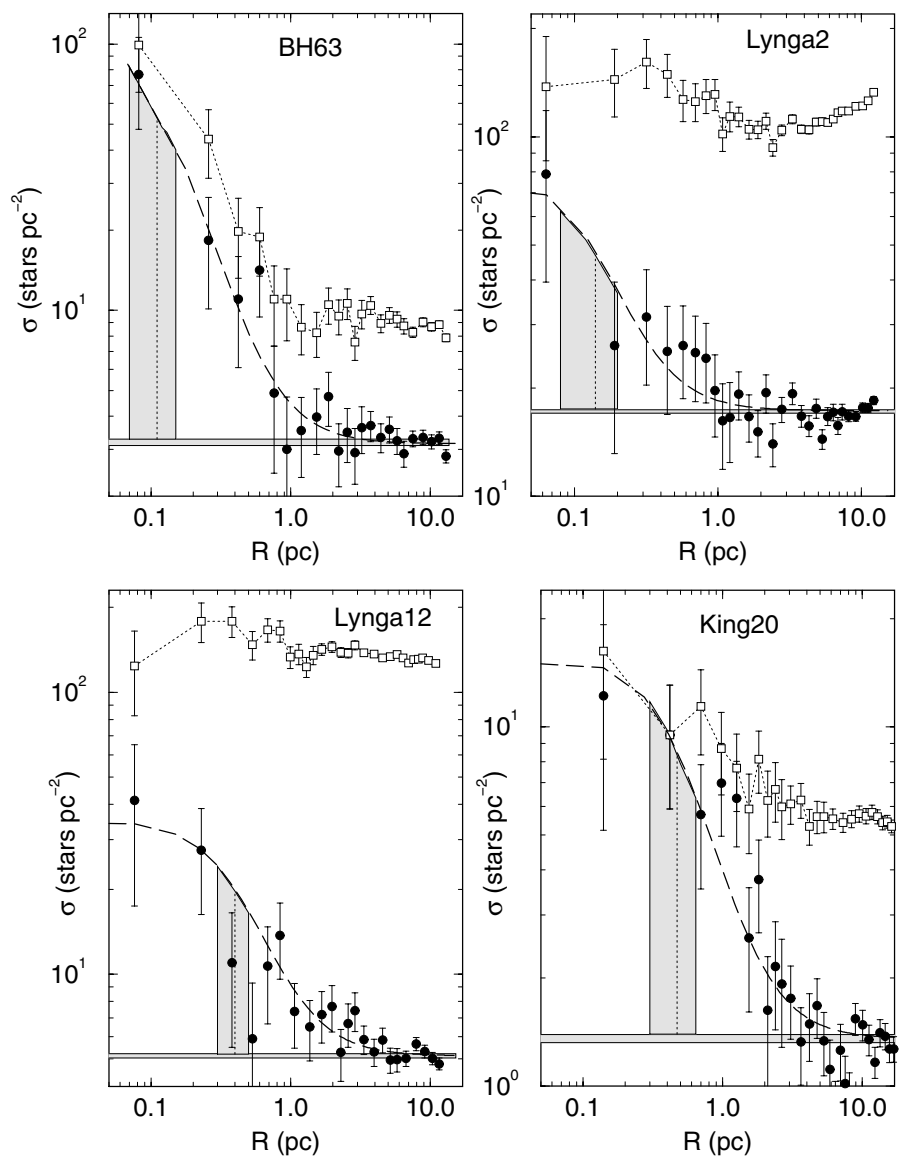

Fig. 5. Stellar radial density profiles. Filled circles: colour-magnitude filtered RDP. Dashed line: best-fit two-parameter King profile. Horizontal shaded region: residual stellar background level measured in the colour-magnitude filtered offset field. Vertical dotted line: bestfit King core radius. Vertical shaded region: standard deviation of the core radius. For comparison purposes the empty squares show the RDPs prior to the C-MF, where the actual stellar background level can be seen.

We also show in Fig. 5 the observed RDPs produced with photometry prior to the use of C-MF. Clearly, the filtered profiles present less fluctuations and go deeper into the cluster structure than the observed ones. In particular, the observed profiles tend to underestimate the cluster's extension. As expected, the C-MF produced residual background levels much lower than the observed ones, especially for Lyngå 2 and Lyngå 12. Besides, CMFs have proven to be essential to unveil the cluster centroid especially for faint OCs such as those in the present sample.

Structural parameters were derived by fitting the C-MF RDPs with the two-parameter King (1966a) surface density profile, which describes the central and intermediate regions of normal clusters (King 1966b; Trager et al. 1995). The fits were performed using a nonlinear least-squares fit routine that uses errors as weights. To minimise degrees of freedom in the fit the background level $\left(\sigma_{\mathrm{bg}}\right)$ was kept constant, corresponding to the residual values measured in the corresponding offset fields (Sect. 3.1). Parameters derived from the fit are the King central density of stars $\left(\sigma_{0 \mathrm{~K}}\right)$ and core radius $\left(R_{\text {core }}\right)$. The resulting parameters are given in Table 3 and the best-fit solutions are superimposed on the C-MF RDPs (Fig. 5). A limiting radius $\left(R_{\lim }\right)$ of a cluster can be estimated by considering the fluctuations of the RDPs with respect to the residual background. $R_{\text {lim }}$ describes where the RDP merges into the background and, for practical purposes most of the cluster stars are contained within $R_{\lim }$. For comparison purposes Table 3 provides parameters in angular and absolute units. Probably because of different methods and data sets the present values of $R_{\text {core }}$ and $R_{\lim }$ for Lyngå 2 correspond to about $1 / 9$ and $1 / 2$ of those given in Kharchenko et al. (2005). We verified that the centroid in both works is virtually coincident. The difference may be attributed to their brighter limits (Kharchenko et al. 2004) producing shallower profiles for this faint cluster.

Compared to other OCs (Sect. 5; Nilakshi 2002; Tadross et al. 2002), the present clusters are small both in core and limiting radii. This is particularly true for Lyngå 2 and BH 63 .

Within uncertainties, King profiles provide a good analytical representation of the stellar RDPs of Lyngå 12 and King 20, from the external parts to the core. However, because of the small size of BH 63 and Lyngå 2 (and the larger distance of BH 63) their RDPs do not reach much into the core. In BH 63 (and to a lesser extent Lyngå 2) the RDP seems to increase almost linearly (in a $\log$ - $\log$ scale) with decreasing distance to the center with no evidence of the King-like core turnover. Consequently, we cannot compare the actual stellar density profiles of BH 63 and Lyngå 2 inside the core with the King profile. Two interpretations are possible. Either the very central region was not accessed or the linear increase points to a post-core collapse structure as those observed by Trager et al. (1995) for several post-core collapse globular clusters.

Since it follows from an isothermal (virialized) sphere, the close similarity of a cluster's stellar RDP with a King profile suggests that the internal structure (particularly the core) has reached some important level of energy equipartition at the cluster age. We return to this point in Sect. 5.

\section{Luminosity and mass functions}

The 2MASS quality photometry along with the methods presented in Bonatto \& Bica (2005, and references therein) allows one to derive luminosity and mass functions $\left(\phi(m)=\frac{\mathrm{d} N}{\mathrm{~d} m}\right)$ for the core, halo and overall (i.e. the whole cluster) regions for the present faint clusters.

In all clusters, the resulting faint-magnitude limit of the MS stars, isolated with the C-MFs (Fig. 3), is brighter than that of the $99.9 \%$ Completeness Limit (Sect. 3). To take the residual field-star contamination into account, we build LFs for each cluster region and offset field separately. $J, H$ and $K_{\mathrm{s}}$ LFs are built by counting stars in magnitude bins from the respective faint magnitude limit to the turn-off, for cluster and offset field regions. Magnitude bins are wider in the upper MS than in the lower MS to avoid undersampling near the turn-off and oversampling at the faint limit. Corrections are made for different solid angles between offset field and cluster regions. Cluster LFs are obtained by subtracting the offset-field LFs. They are transformed into MFs using the mass-luminosity relations obtained from the respective Padova isochrones and observed distance modulii (Sect. 3). These procedures are applied independently to the three 2MASS bands. Because of differences among the $J, H$, and $K_{\mathrm{s}}$ mass-luminosity relations, the respective MFs are sampled in different mass values. Consequently, the final MF is simply a superposition of the $J, H$ and $K_{\mathrm{S}}$ MFs. Detected MS mass ranges are $1.5 \leq m\left(M_{\odot}\right) \leq 2.3,0.9 \leq m\left(M_{\odot}\right) \leq 5.4$, $0.8 \leq m\left(M_{\odot}\right) \leq 2.5$ and $1.1 \leq m\left(M_{\odot}\right) \leq 3.8$, respectively for BH 63, Lyngå 2, Lyngå 12 and King 20. Sub-solar mass stars are expected to populate the MS of OCs older than $\approx 100 \mathrm{Myr}$, however, they cannot be detected with 2MASS because of the increasing fraction of field-star contamination in that mass range 
Table 3. Structural parameters.

\begin{tabular}{lccccccccc}
\hline \hline Cluster & $\begin{array}{c}1^{\prime} \\
(\mathrm{pc})\end{array}$ & $\begin{array}{c}\sigma_{\mathrm{bg}} \\
\left(\mathrm{stars}^{\prime-2}\right)\end{array}$ & $\begin{array}{c}\sigma_{0 \mathrm{~K}} \\
\left(\mathrm{stars}^{\prime-2}\right)\end{array}$ & $\begin{array}{c}R_{\text {core }} \\
\left(^{\prime}\right)\end{array}$ & $\begin{array}{c}R_{\lim } \\
\left(^{\prime}\right)\end{array}$ & $\begin{array}{c}\sigma_{\mathrm{bg}} \\
\left(\mathrm{stars} \mathrm{pc}^{-2}\right)\end{array}$ & $\begin{array}{c}\sigma_{0 \mathrm{~K}} \\
\left(\mathrm{stars} \mathrm{pc}^{-2}\right)\end{array}$ & $\begin{array}{c}R_{\text {core }} \\
(\mathrm{pc})\end{array}$ & $\begin{array}{c}R_{\lim } \\
(\mathrm{pc})\end{array}$ \\
$(1)$ & $(2)$ & $(3)$ & $(4)$ & $(5)$ & $(6)$ & $(7)$ & $(8)$ & $(9)$ & $(10)$ \\
\hline BH 63 & 0.680 & $1.46 \pm 0.03$ & $52 \pm 29$ & $0.16 \pm 0.06$ & $2.4 \pm 0.5$ & $3.1 \pm 0.1$ & $112 \pm 63$ & $0.11 \pm 0.04$ & $1.6 \pm 0.3$ \\
Lyngå 2 & 0.254 & $1.12 \pm 0.01$ & $4.1 \pm 2.2$ & $0.54 \pm 0.24$ & $5.5 \pm 0.8$ & $17.3 \pm 0.2$ & $63 \pm 34$ & $0.14 \pm 0.06$ & $1.4 \pm 0.2$ \\
Lyngå 12 & 0.304 & $0.48 \pm 0.01$ & $2.8 \pm 1.2$ & $1.30 \pm 0.37$ & $13 \pm 2$ & $4.8 \pm 0.1$ & $30 \pm 13$ & $0.40 \pm 0.10$ & $3.9 \pm 0.6$ \\
King 20 & 0.560 & $0.43 \pm 0.01$ & $4.5 \pm 2.6$ & $0.84 \pm 0.33$ & $7 \pm 1$ & $1.4 \pm 0.1$ & $14 \pm 8$ & $0.47 \pm 0.18$ & $3.9 \pm 0.6$ \\
\hline
\end{tabular}

Table notes: Col. 2: arcmin to parsec scale. We express King profile as $\sigma(R)=\sigma_{\mathrm{bg}}+\sigma_{0 \mathrm{~K}} /\left(1+\left(R / R_{\mathrm{core}}\right)^{2}\right)$. To minimise degrees of freedom in the fit $\sigma_{\mathrm{bg}}$ was kept fixed (measured in the respective offset fields) while $\sigma_{0 \mathrm{~K}}$ and $R_{\text {core }}$ were allowed to vary. Note that $\sigma_{0 \mathrm{~K}}$ and $\sigma_{\mathrm{bg}}$ represent residual densities of stars, measured on the C-MF photometry.
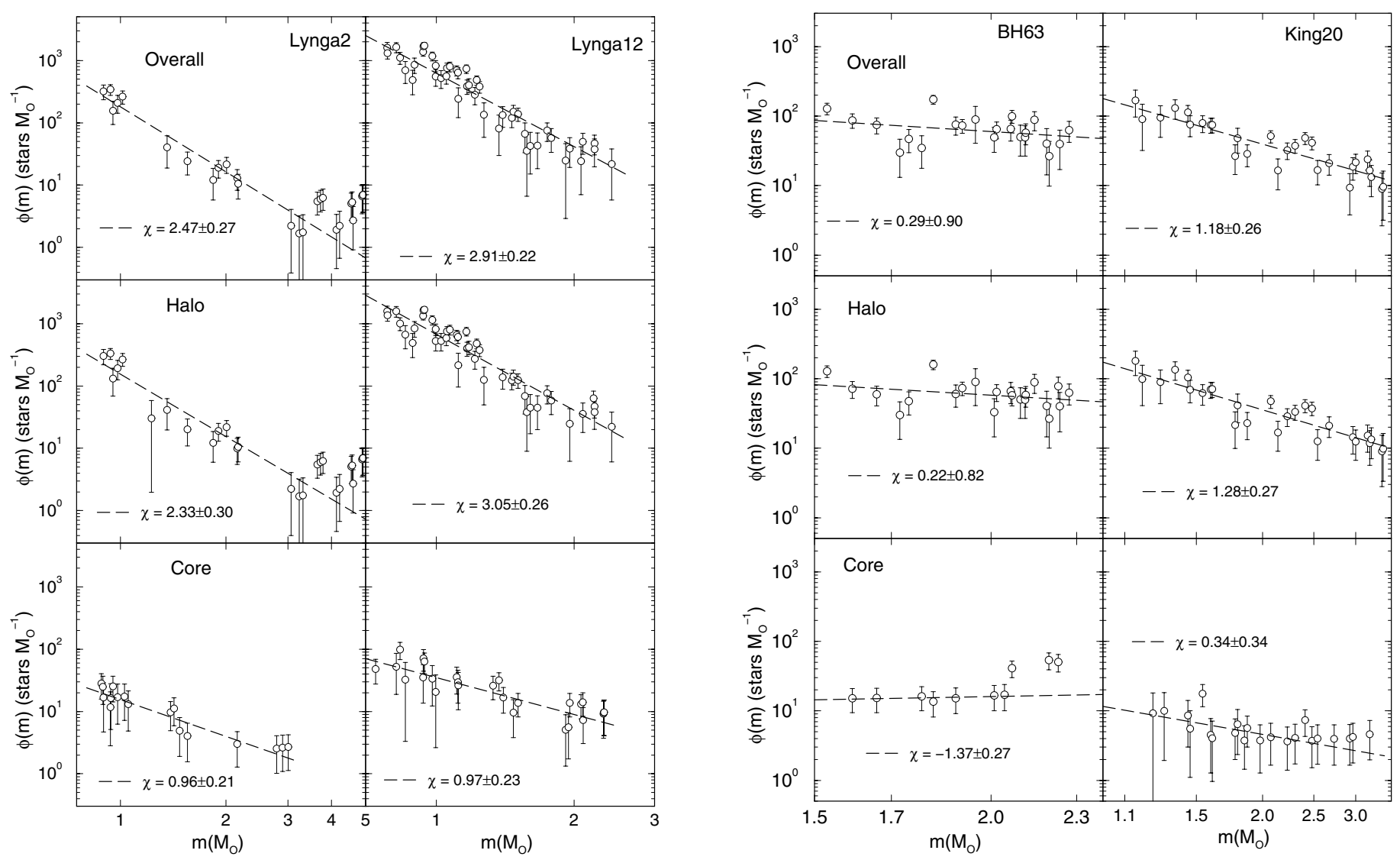

Fig. 6. Colour-magnitude filtered mass functions (empty circles) fitted with the function $\phi(m) \propto m^{-(1+\chi)}$ (dashed line). Top, middle and bottom panels contain the MFs of the overall cluster, halo and core regions.

(right panels of Fig. 3). The observed MS range was fitted with the function $\phi(m) \propto m^{-(1+\chi)}$.

The overall, halo and core MFs of Lyngå 2 and Lyngå 12 are shown in the left panels of Fig. 6, while those of BH 63 and King 20 are in the right panels. The MF slopes are given in Col. 4 of Table 4.

A common feature among the OCs is the flat core MF, particularly in BH 63; the overall MF of this OC is much flatter than Salpeter's (1955) IMF ( $\chi=1.35)$. Lyngå 2 and Lyngå 12 have halo and overall MFs steeper than Salpeter (Sect. 5).

\subsection{Cluster mass}

Parameters derived from the core, halo and overall MFs of the target clusters are given in Table 4 . The number of evolved stars
(Col. 2) in each region was obtained by integration of the respective field-star subtracted LF for stars brighter than the turnoff. Multiplying this number by the mass at the turn-off yields an estimate of the mass stored in evolved stars (Col. 3). The observed number of MS stars and corresponding mass (Cols. 5 and 6 , respectively) were derived by integrating the MFs in the mass ranges.

To estimate the total mass locked up in stars we extrapolate the observed MFs down to the H-burning mass limit $\left(0.08 M_{\odot}\right)$ since these stars are expected to be present in the MS of OCs older than 90 Myr. In the mass range below the present detection threshold (Table 4) we base the extrapolation on Kroupa's (2001) universal Initial Mass Function (IMF), in which $\chi=0.3 \pm 0.5$ for the range $0.08 \leq m\left(M_{\odot}\right) \leq 0.5$ and $\chi=1.3 \pm 0.3$ for $0.5 \leq$ $m\left(M_{\odot}\right) \leq 1.0$. We remark that in the cases where our MF slopes are flatter than or similar (within uncertainties) to Kroupa's, we 
Table 4. Parameters related to mass functions and dynamical states.

\begin{tabular}{|c|c|c|c|c|c|c|c|c|c|c|}
\hline \multirow[b]{2}{*}{ Region } & \multicolumn{2}{|c|}{ Evolved } & \multicolumn{3}{|c|}{ Observed MS } & \multicolumn{5}{|c|}{ Evolved + Extrapolated MS } \\
\hline & $\begin{array}{c}N^{*} \\
\text { (stars) }\end{array}$ & $\begin{array}{c}m \\
\left(M_{\odot}\right)\end{array}$ & $\chi$ & $\begin{array}{c}N^{*} \\
\text { (stars) }\end{array}$ & $\begin{array}{l}m_{\mathrm{obs}} \\
\left(M_{\odot}\right)\end{array}$ & $\begin{array}{c}N^{*} \\
\left(10^{2} \text { stars }\right)\end{array}$ & $\begin{array}{c}m \\
\left(10^{2} M_{\odot}\right)\end{array}$ & $\begin{array}{c}\sigma \\
\left(M_{\odot} \mathrm{pc}^{-2}\right)\end{array}$ & $\begin{array}{c}\rho \\
\left(M_{\odot} \mathrm{pc}^{-3}\right)\end{array}$ & $\tau$ \\
\hline (1) & (2) & (3) & (4) & (5) & (6) & (7) & (8) & (9) & (10) & (11) \\
\hline & \multicolumn{10}{|c|}{ BH $63-\mathrm{MS}: 1.5 \leq m\left(M_{\odot}\right) \leq 2.3-\mathrm{Age}=700 \pm 100 \mathrm{Myr}$} \\
\hline Core & - & - & $-1.37 \pm 0.27$ & $12 \pm 3$ & $22 \pm 5$ & $0.3 \pm 0.1$ & $0.4 \pm 0.1$ & $960 \pm 150$ & $6500 \pm 1000$ & $19200 \pm 7690$ \\
\hline Halo & $2 \pm 1$ & $5 \pm 2$ & $0.22 \pm 0.82$ & $46 \pm 33$ & $87 \pm 62$ & $5.6 \pm 5.3$ & $3.1 \pm 1.4$ & $38 \pm 17$ & $18 \pm 8$ & - \\
\hline \multirow[t]{2}{*}{ Overall } & $2 \pm 1$ & $5 \pm 2$ & $0.29 \pm 0.90$ & $48 \pm 25$ & $90 \pm 47$ & $6.5 \pm 6.0$ & $3.4 \pm 1.2$ & $42 \pm 15$ & $20 \pm 7$ & $108 \pm 88$ \\
\hline & \multicolumn{10}{|c|}{ Lyngå $2-$ MS: $0.9 \leq m\left(M_{\odot}\right) \leq 5.4-$ Age $=90 \pm 10 \mathrm{Myr}$} \\
\hline Core & - & - & $0.96 \pm 0.21$ & $12 \pm 2$ & $19 \pm 3$ & $1.0 \pm 0.7$ & $0.4 \pm 0.1$ & $612 \pm 182$ & $3060 \pm 909$ & $697 \pm 475$ \\
\hline Halo & $1 \pm 1$ & $5 \pm 5$ & $2.33 \pm 0.30$ & $83 \pm 16$ & $119 \pm 23$ & $13 \pm 10$ & $4.6 \pm 1.9$ & $75 \pm 31$ & $40 \pm 16$ & - \\
\hline \multirow[t]{2}{*}{ Overall } & $1 \pm 1$ & $5 \pm 5$ & $2.47 \pm 0.27$ & $94 \pm 17$ & $136 \pm 27$ & $15 \pm 11$ & $5.3 \pm 2.2$ & $86 \pm 36$ & $46 \pm 19$ & $7.7 \pm 5.3$ \\
\hline & \multicolumn{10}{|c|}{ Lyngå $12-$ MS: $0.8 \leq m\left(M_{\odot}\right) \leq 2.5-\mathrm{Age}=560 \pm 100 \mathrm{Myr}$} \\
\hline Core & $1 \pm 1$ & $3 \pm 3$ & $0.97 \pm 0.23$ & $33 \pm 3$ & $42 \pm 5$ & $2.2 \pm 1.6$ & $1.0 \pm 0.3$ & $187 \pm 58$ & $350 \pm 110$ & $846 \pm 534$ \\
\hline Halo & $7 \pm 3$ & $18 \pm 8$ & $3.05 \pm 0.26$ & $539 \pm 37$ & $556 \pm 40$ & $83 \pm 65$ & $25 \pm 12$ & $54 \pm 25$ & $10 \pm 5$ & - \\
\hline \multirow[t]{2}{*}{ Overall } & $8 \pm 3$ & $20 \pm 8$ & $2.91 \pm 0.22$ & $500 \pm 31$ & $521 \pm 34$ & $74 \pm 58$ & $23 \pm 11$ & $48 \pm 22$ & $9.2 \pm 0.4$ & $4.3 \pm 3.1$ \\
\hline & \multicolumn{10}{|c|}{ King $20-$ MS: $1.1 \leq m\left(M_{\odot}\right) \leq 3.8-$ Age $=200 \pm 20 \mathrm{Myr}$} \\
\hline Core & $1 \pm 1$ & $3 \pm 3$ & $0.34 \pm 0.34$ & $10 \pm 3$ & $20 \pm 6$ & $0.6 \pm 0.2$ & $0.4 \pm 0.1$ & $56 \pm 12$ & $90 \pm 20$ & $735 \pm 365$ \\
\hline Halo & $6 \pm 2$ & $20 \pm 7$ & $1.28 \pm 0.27$ & $79 \pm 20$ & $135 \pm 40$ & $13 \pm 9$ & $5.5 \pm 1.9$ & $12 \pm 4$ & $2.2 \pm 0.7$ & - \\
\hline Overall & $7 \pm 3$ & $24 \pm 10$ & $1.18 \pm 0.26$ & $91 \pm 22$ & $157 \pm 44$ & $13 \pm 9$ & $5.6 \pm 1.8$ & $12 \pm 4$ & $2.2 \pm 0.7$ & $7.0 \pm 4.5$ \\
\hline
\end{tabular}

Table notes: Col. 6: stellar mass of the observed MS; Col. 8: mass extrapolated to $0.08 M_{\odot}$; Col. 11: dynamical-evolution parameter $\tau=$ age $/ t_{\text {rel }}$.

directly use the former slopes, e.g. the three MFs of BH 63; for the core MF of Lyngå 2 we use our slope in the range $0.5 \leq$ $m\left(M_{\odot}\right) \leq 0.9$, and Kroupa's for $0.08 \leq m\left(M_{\odot}\right) \leq 0.5$.

The total (extrapolated MS + evolved) values of number, mass, projected and volume densities are given in Cols. 7 to 10 of Table 4.

\section{Diagnostic-diagrams of dynamical states}

The King-like RDPs (Fig. 5) and the change in MF slope from core to halo (Fig. 6) may be a consequence of dynamical evolution in the present OCs, particularly in the core. At this point it is interesting to see how these faint clusters compare with a set of nearby OCs in terms of structure and dynamical state.

Bonatto \& Bica (2005) derived a set of parameters related to the structure and dynamical state of nearby OCs with ages in the range $70-7000 \mathrm{Myr}$ and masses within $400-5300 M_{\odot}$, following most of the present methodology. To the original sample were added the very young OC (age $\sim 1.3 \mathrm{Myr}$ ) NGC 6611 (Bonatto et al. 2006) and the young one (age $\sim 14 \mathrm{Myr}$ ) NGC 4755 (Bonatto et al. 2006b). The evolutionary parameter $\tau=$ age $/ t_{\text {rel }}$ (Col. 11 of Table 4), where $t_{\text {rel }}$ is the relaxation time, was found to be a good tracer of dynamical states. In general terms, $\tau$ is a measure of the dynamical age of a cluster as a whole or of a cluster's internal region. In this sense, effects of dynamical processes such as mass segregation and low-mass star evaporation on MF slopes are expected to become more conspicuous with increasing $\tau$. In particular, Bonatto \& Bica (2005) observed that significant flattening in core and overall MFs due to dynamical effects occurs for $\tau_{\text {core }} \geq 100$ and $\tau_{\text {overall }} \geq 7$, respectively. The difference in core and overall $\tau$ threshold is associated with the different average physical conditions in both regions, such as density and gravitational potential, which in turn affect $t_{\text {rel }}$. Further discussions and details on parameter correlation in the reference sample are in Bonatto \& Bica (2005).

Core and overall radii in the reference sample are related by the equation $R_{\lim } \propto R_{\mathrm{core}}^{(0.9 \pm 0.1)}$. Despite the small sizes of $\mathrm{BH} 63$
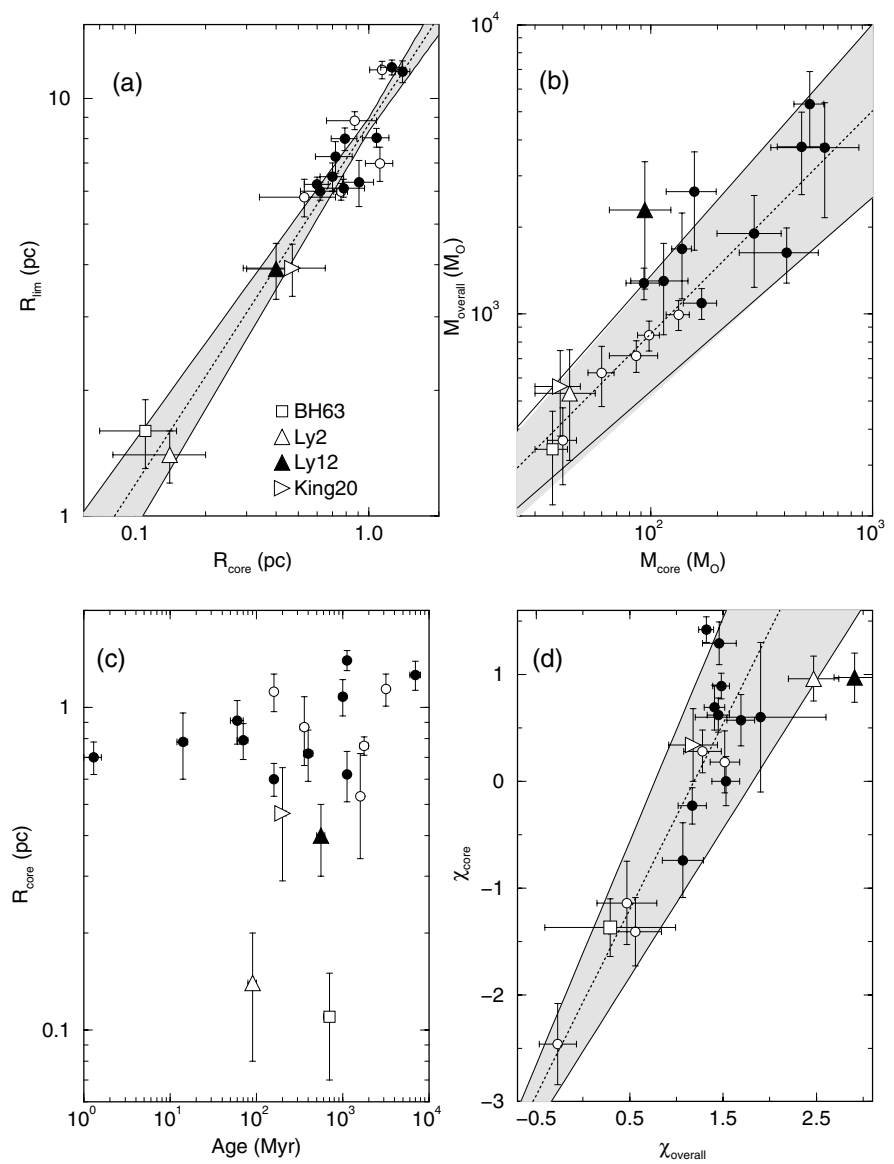

Fig. 7. Relations involving core and limiting radii (panel a)), core and overall mass (panel b)), core radius and cluster age (panel c)), and core and overall MF slope (panel d)). Filled symbols: clusters more massive than $1000 M_{\odot}$ (Bonatto \& Bica 2005). Open symbols: $m<1000 M_{\odot}$. Dotted lines: least-squares fits to the reference clusters. Shaded areas: $1 \sigma$ borders of the least-squares fits. 

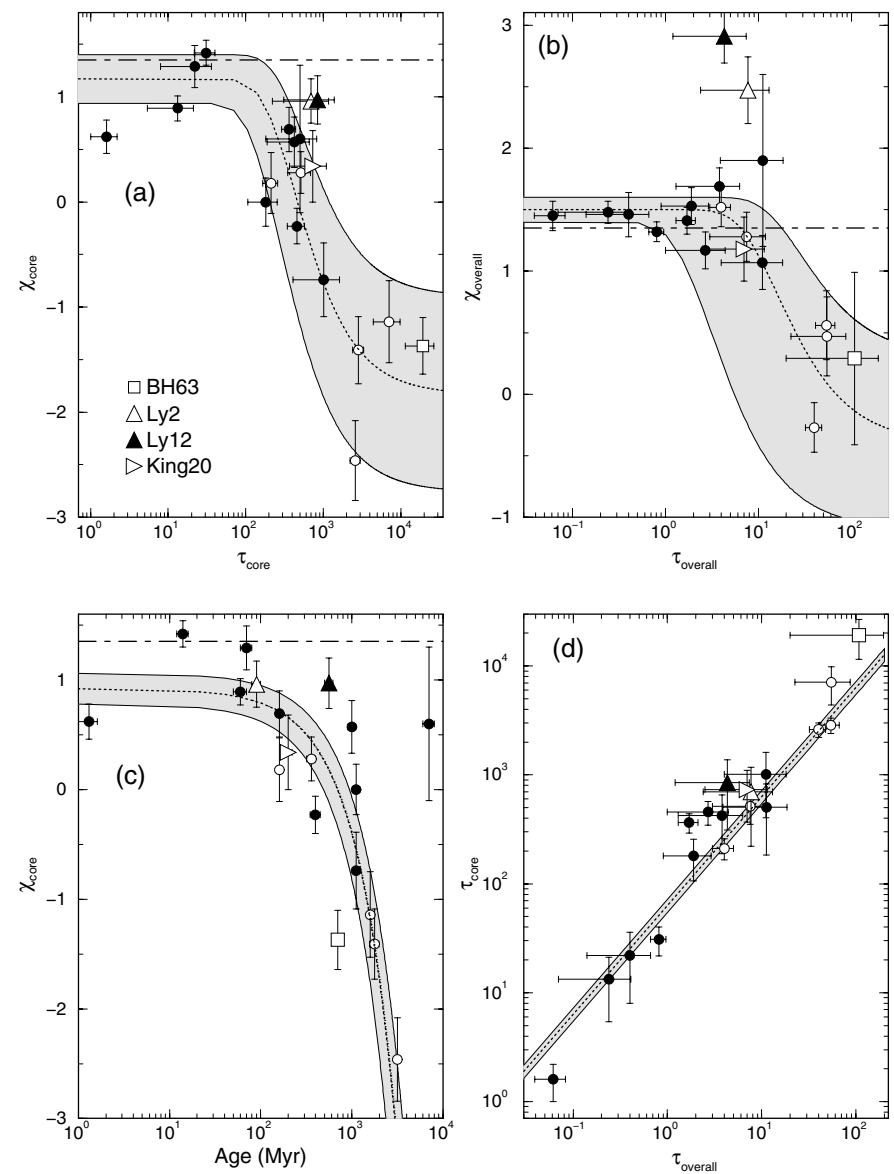

Fig. 8. Relations involving MF slope and evolutionary parameter for the core (panel a)) and overall cluster (panel b)); core MF slope and cluster age (panel c)); and core and overall evolutionary parameter (panel d)). The dot-dash line in panels a), b) and c) indicates the Salpeter IMF slope value. Symbols as in Fig. 7.

and Lyngå 2, the present OCs have $R_{\text {lim }}$ and $R_{\text {core }}$ consistent with that relation at the $1 \sigma$ level (panel a of Fig. 7). The same occurs for the core and overall (extrapolated) mass $\left(m_{\text {overall }} \propto m_{\text {core }}^{(0.8 \pm 0.1)}\right.$, panel $b$ ). Within uncertainties, both slopes are the same which is probably a consequence of scaling, in the sense that on average massive clusters tend to be big. About $2 / 3$ of the reference sample suggests a trend of increasing core radius with age (panel c), while the remaining ones - Lyngå 2 and BH 63 in particular - appear to follow a sequence of decreasing $R_{\text {core }}$ with age. Except for the steep overall MF of Lyngå 12, the remaining OCs have MF slopes consistent with those of the reference sample (panel d).

Relations involving MF slope and dynamical-evolution parameter (Fig. 8) can be used for inferences on dynamical states. Panels (a) and (b) suggest that both the core and overall MF slopes decay with the evolutionary parameter according to $\chi(\tau)=\chi_{\mathrm{o}}-\chi_{1} \times \mathrm{e}^{-\left(\frac{\tau_{0}}{\tau}\right)}$ (Bonatto \& Bica 2005), where $\chi_{\mathrm{o}}$ represents the MF slope at cluster birth, while $\chi_{1}$ gives the amount of slope flattening for advanced evolutionary states. Panel (c) suggests a systematic flattening of the core MF slope with cluster age, particularly for the less-massive OCs ${ }^{7}$. Except for NGC 188, the age dependence of the core MF slope can be parameterised

7 The deviant object is the $\approx 7 \mathrm{Gyr}$, massive OC NGC 188 whose orbit avoids the inner regions of the Galaxy for most of the time (Bonatto et al. 2005, and references therein). by the linear-decay function $\chi(t)=\chi_{\mathrm{o}}-t / t_{\mathrm{f}}$, where $\chi_{\mathrm{o}}$ may be interpreted as the core MF slope in the early phases of the cluster, and $t_{\mathrm{f}}$ is the flattening time scale associated mostly with mass segregation. We find $t_{\mathrm{f}}=775 \pm 130 \mathrm{Myr}$ and $\chi_{\mathrm{o}}=0.92 \pm 0.14$, a value significantly flatter than that of Salpeter IMF. Core and overall evolutionary parameters (panel d) are related by $\tau_{\text {overall }} \propto$ $\tau_{\text {core }}^{(0.9 \pm 0.1)}$. Further discussions are in Bonatto \& Bica (2005).

As shown in all panels of Fig. 8, BH 63 presents clear signs of accelerated dynamical evolution, particularly in the core. With flat MF slopes and large dynamical evolution parameters, the core and overall regions of $\mathrm{BH} 63$ appear to be extreme cases of OC dynamical evolution (especially in panels a and b). One possibility is that interaction with the nebula FeSt 1-108 (Sect. 2) may have accelerated its dynamical evolution. Although to a lesser degree, similar evidence for the core and overall regions of King 20 is provided in all panels. The cores of Lyngå 2 and Lyngå 12 show marginal evidence of dynamical evolution (flat MF slopes).

\section{Concluding remarks}

In this paper, we derived photometric and structural parameters of the faint open clusters BH 63, Lyngå 2, Lyngå 12, and King 20 with $J, H$ and $K_{\mathrm{s}}$ 2MASS photometry, restricted to stars with observational uncertainties $\epsilon_{J, H, K_{\mathrm{S}}}<0.2 \mathrm{mag}$. BH 63 is significantly more reddened than the others, with $E(B-V) \approx 1.9$. Distance from the Sun ranges from $d_{\odot} \approx 0.9 \mathrm{kpc}$ (Lyngå2) to $d_{\odot} \approx 2.3 \mathrm{kpc}$ (BH 63). Lyngå 2 and Lyngå 12 are located inside the Solar circle.

The use of C-MFs allowed us to obtain accurate parameters for such faint clusters including mass function slopes for the core and halo. In particular, radial density profiles built with C-MF photometry are better defined and yield more constrained structural parameters than the observed ones. The RDPs of Lyngå 12 and King 20 are well-represented by a King profile. Lyngå 2 and BH 63 appear to be very small, with core and limiting radii of $\approx 0.12$ pc and $\approx 1.5$ pc. However, they fit in the small core and limiting radii tail of the OC distribution. Lynga 12 and King 20 have $R_{\text {core }} \approx 0.43 \mathrm{pc}$ and $R_{\lim } \approx 3.9 \mathrm{pc}$.

The open clusters of the present sample have flat core MFs, suggesting dynamically evolved systems, particularly BH 63, which has a flat MF in its halo as well. The total mass locked up in stars (extrapolated down to $0.08 M_{\odot}$ ) ranges from $\approx 340 M_{\odot}$ (BH 63) to $\approx 2300 M_{\odot}$ (Lyngå 12).

We also analysed the clusters with dynamical and structural diagnostic diagrams. BH 63 appears to be an extreme case of OC dynamical evolution both in its core and halo, perhaps due to interaction with the nebula FeSt 1-108. King 20 shows signs of dynamical evolution as well, but to a lesser degree. The cores of Lyngå 2 and Lyngå 12 present marginal evidence of dynamical evolution.

The present work shows that it is possible to explore faint OCs with 2MASS photometry, provided care is taken to identify probable member stars (to better define the cluster sequence on CMDs) and discard stars with discordant colour (for more intrinsic cluster RDPs and MFs). The use of field-star decontamination and colour-magnitude filters on the fields of $\mathrm{BH} 63$, Lyngå 2, Lyngå 12 and King 20 allowed us to obtain good results for the fundamental, structural and dynamical-evolution related parameters.

Acknowledgements. We thank the anonymous referee for helpful suggestions. This publication makes use of data products from the Two Micron All Sky Survey, which is a joint project of the University of Massachusetts and the 
Infrared Processing and Analysis Center/California Institute of Technology, funded by the National Aeronautics and Space Administration and the National Science Foundation. This research has made use of the WEBDA database, operated at the Institute for Astronomy of the University of Vienna. CB and $\mathrm{EB}$ acknowledge support from the Brazilian Institution $\mathrm{CNPq}$, and $\mathrm{RB}$ a $\mathrm{CNPq} / \mathrm{UFRGS}$ PIBIC fellowship.

\section{References}

Alter, G., Balazs, B., Ruprecht, J., \& Vanysek, J. 1970, in Catalogue of star clusters and associations 2nd edn., ed. G. Alter, B. Balazs, \& J. Ruprecht (Budapest: Akademiai Kiado)

Bessel, M. S., \& Brett, J. M. 1988, PASP, 100, 1134

Bica, E., \& Bonatto, C. 2005, A\&A, 443, 465

Bica, E., Bonatto, C., \& Dutra, C. M. 2003, A\&A, 405, 991

Bica, E., Bonatto, C., \& Dutra, C. M. 2004, A\&A, 422, 555

Bica, E., Bonatto, C., Barbuy, B., \& Ortolani, S. 2006, A\&A, 450, 105

Bonatto, C., \& Bica, E. 2003, A\&A, 405, 525

Bonatto, C., \& Bica, E. 2005, A\&A, 437, 483

Bonatto, C., \& Bica, E. 2006, A\&A, 455, 931

Bonatto, C., Bica, E., \& Girardi, L. 2004a, A\&A, 415, 571

Bonatto, C., Bica, E., \& Pavani, D. B. 2004b, A\&A, 427, 485

Bonatto, C., Bica, E., \& Santos Jr., J. F. C. 2005, A\&A, 433, 917

Bonatto, C., Santos, Jr., J. F. C., \& Bica, E. 2006, A\&A, 445, 567

Bonatto, C., Kerber, L. O., Santiago, B. X., \& Bica, E. 2006a, A\&A, 446, 121

Bonatto, C., Bica, E., Ortolani, S., \& Barbuy, B. 2006b, A\&A, 453, 121

Dias, W. S., Alessi, B. S., Moitinho, A., \& Lépine, J. R. D. 2002, A\&A, 389, 871

Dutra, C. M., \& Bica, E. 2002, A\&A, 381, 631
Dutra, C. M., Santiago, B. X., \& Bica, E. 2002, A\&A, 383, 219

Feitzinger, J. V., \& Stüwe, J. A. 1984, A\&AS, 58, 355

Friel, E. D. 1995, ARA\&A, 33, 38

Girardi, L., Bertelli, G., Bressan, A., et al. 2002, A\&A, 391, 195

Janes, K. A., \& Phelps, R. L. 1994, AJ, 108, 1773

Kharchenko, N. V., Piskunov, A. E., Röser, S., Schilbach, E., \& Scholz, R.-D. 2004, AN, 325, 740

Kharchenko, N. V., Piskunov, A. E., Röser, S., Schilbach, E., \& Scholz, R.-D. 2005, A\&A, 438, 1163

King, I. 1966a, AJ, 71, 64

King, I. 1966b, AJ, 71, 276

Kroupa, P. 2001, MNRAS, 322, 231

Lindoff, U. 1968, ArA, 4, 471

Lyngå, G. 1982, A\&A, 109, 213

Mermilliod, J. C. 1996, in The Origins, Evolution, and Destinies of Binary Stars in Clusters, ed. E. F. Milone, \& J.-C. Mermilliod, ASP Conf. Ser., 90, 475

Nilakshi, S. R., Pandey, A. K., \& Mohan, V. 2002, A\&A, 383, 153

Piskunov, A. E., Kharchenko, N. V., Röser, S., Schilbach, E., \& Scholz, R.-D. 2006, A\&A, 445, 545

Reid, M. J. 1993, ARA\&A, 31, 345

Ruprecht, J. 1966, BAICz, 17, 33

Salpeter, E. 1955, ApJ, 121, 161

Skrutskie, M., Schneider, S. E., Stiening, R., et al. 1997, in The Impact of Large Scale Near-IR Sky Surveys, ed. Garzon et al. (Netherlands: Kluwer), 210, 187

Soares, J. B., \& Bica, E. 2002, A\&A 388, 172

Tadross, A. L., Werner, P., Osman, A., \& Marie, M. 2002, NewAst, 7, 553

Trager, S. C., King, I. R., \& Djorgovski, S. 1995, AJ, 109, 218

van den Bergh, S., \& Hagen, G. L. 1975, AJ, 80, 11 